\title{
The study on experimental method of fluid stress sensitivity of low permeability in tight reservoir
}

\author{
Xiao Lin ${ }^{1} \cdot$ Liu Xue-wei $^{2,3} \cdot$ Shao Long-kan ${ }^{1} \cdot$ Wang Shao-peng ${ }^{1} \cdot$ Xiao Pu-fu ${ }^{4} \cdot$ Zhao Xin-li $^{2,3}$ (C) $\cdot$ Niu Zhong-kun ${ }^{2,3}$
}

Received: 2 January 2021 / Accepted: 12 May 2021 / Published online: 14 June 2021

(c) The Author(s) 2021

\begin{abstract}
According to the industry standard, low permeability and tight reservoir are highly stress-sensitive in laboratory tests. However, this phenomenon has not been effectively confirmed in the field production test. For this reason, a method to measure stress sensitivity by axial loading is proposed. By loading fixed axial stress, the measurement method achieves the effect of equivalent preloading fluid pressure. Meanwhile, the equivalent theoretical curve can be obtained by translating the curve. Through this method, the stress sensitivity of the core with microfracture is studied. According to the results, the stress sensitivity of the cores can be divided into two stages: the first stage is the non-opening stage of microfractures, in which the stress sensitivity is weak; the second stage is the opening stage of microfractures, in which the stress sensitivity increases sharply. When the permeability changes significantly, the corresponding pressure is the microfracture's opening pressure. In addition, if the abscissa of the experimental results corresponds to the field production data, the field stress sensitivity curve of the field reservoir can be obtained. Conventional stress sensitivity test shows that the four cores in the study block exhibit medium to strong stress sensitivity. According to the stress sensitivity curve analysis after curve translation, the stress sensitivity of the reservoir is about $25 \%$ in the elastic production stage, which is classified as weak stress sensitivity. This conclusion can effectively support the phenomenon of the unobvious stress sensitivity in the field production of low permeability and tight reservoirs.
\end{abstract}

Keywords Stress sensitivity $\cdot$ Experimental method $\cdot$ Axial stress $\cdot$ Microfractures $\cdot$ Fracture re-open pressure

\section{Introduction}

Reservoir stress sensitivity refers to the phenomenon that reservoir permeability changes due to the influence of reservoir stress conditions (Cao et al. 2019; Chen et al. 2015; Xiao et al. 2016). Because of the stress sensitivity of low

Liu Xue-wei

ucaszxl@163.com

$\triangle$ Zhao Xin-li

zhaoxinli17@mails.ucas.edu.cn

1 North China Oil and Gas Branch, SINOPEC, Zhengzhou 450006, P. R. China

2 Research Institute of Petroleum Exploration and Development, PetroChina, Beijing 100083, P. R. China

3 Institute of Porous Flow and Fluid Mechanics, University of Chinese Academy of Sciences, Langfang 065007, P.R. China

4 Research Institute of Petroleum Exploration and Development, SINOPEC, Beijing 100083, P. R. China permeability and tight reservoir, more and more attention has been paid to the study of reservoir stress sensitivity, especially in the field of low permeability and micro-fracture developed reservoirs. A lot of literature has been published in recent years (Zhang et al. 2014, 2019; Dou et al. 2016; McKee et al. 1988; Meng et al. 2015; Liang et al. 2016; Liu et al. 2018; Boer et al. 1992; Tang et al. 2013; Li et al. 2009; Meng et al. 2015).

At present, the stress sensitivity measurement standard adopted by the oil industry is SY / T5358-2010 (National Energy Administration 2010). According to the standard, in the process of oil and gas exploitation, the pore pressure decreases with the production of internal fluid, which breaks the original stress balance state and results in elastic or plastic deformation of the rock. Hence, the changes in pore structure and pore volume also influence the seepage capacity of the porous medium. The purpose of the stress sensitivity test is to understand the deformation of the pore and throat, the process by which fracture closes or opens 
as the net stress changes, which leads to the change of rock seepage capacity.

The standard of SY / T5358-2010 suggested experimental methods include two types: one is the variable confining pressure test method, the other is the variable backpressure test method. The way of confining pressure change is to open the outlet of the core to the atmosphere while remain the inlet pressure of the core unchanged during the experiment. The net stress changes with the changing confining pressure. The way of the variable backpressure method is to simulate the overburden pressure with constant confining pressure during the experiment and add the backpressure at the outlet of the core. Under a certain displacement speed, the backpressure is changed to adjust the pore pressure. The change of net stress of rock particles is realized by fluid pressure. The standard of SY / T5358-2010 recommends that the stress sensitivity test be conducted using gas, kerosene, formation water, or potassium chloride brine.

Yan et al. (2018) found that axial stress loading and confining pressure loading speed will affect the experimental results of stress sensitivity in the variable confining pressure test. Li (2009) believed that low permeability and tight reservoirs were not highly stress-sensitive. He (2016) proposed that the experimental results of confining pressure method and variable back pressure method had a corresponding relationship and could be converted to each other. After studying the stress sensitivity of low permeability cores, Sheng et al. (2016) proposed that the in-situ stress sensitivity is weaker than the conventional experimental value. Chen et al. (2015) applied the pulse attenuation permeability measurement method to stress sensitivity measurement because it is difficult to measure the permeability of tight cores. Zhao et al. (2020) evaluated the conventional stress sensitivity by using the cores with micro-fractures and matrix cores and believed that the stress sensitivity of the core with microfractures was stronger than that of the matrix core.

Through literature research, it can be found that there is a certain gap between the existing stress sensitivity test methods and the field application. At present, the research on the stress sensitivity test methods is mainly to solve the measurement problems presented in the existing methods. Although some literature mentions the significant influence caused by axial stress and in-situ conditions on the experimental results, it is still in the qualitative stage.

The author thinks that the current experimental standard provides operational experimental methods for measuring reservoir stress sensitivity. However, besides its difficulty and limitation of development in oilfield application, the two experimental methods cannot effectively characterize the actual stress sensitivity characteristics of the reservoir. Therefore, based on the analysis of the limitations of the two experimental methods, an improved experimental method for measuring reservoir stress sensitivity measurement is proposed and the experimental results of the improved method are analyzed.

The note of the standard emphasizes that the plastic deformation of loose sandstone can pose limitations on the standard method. This paper mainly focuses on low permeability and tight reservoir. Since the focus of this paper, the low permeability and tight reservoir, can be considered as elastic deformation, the influence of plastic deformation is negligible.

\section{Analysis of the limitations of standard experimental methods}

\section{Limitation analysis of variable confining pressure test method}

The stress diagram of rock particles in pores is shown in Fig. 1. There are two ways to change the net stress of rock: The first way is the stress propagated by solid particles, which generally results from the weight of overlying strata and the compressive stress of side rock. In this paper, this type of stress is called solid source conduction stress. The second way results from reservoir fluid pressure. The two stress sources are independent and do not interfere with each other without deformation. The difference between the two stress sources is the net stress of the particle. Without consideration of direction, the net stress on the particle can be expressed as follows:

$\delta_{e}=\delta-p_{l} \varphi$

where $\delta$ is stress, e is effective, $p_{1}$ is fluid pressure and $\varphi$ is surface porosity or porosity.

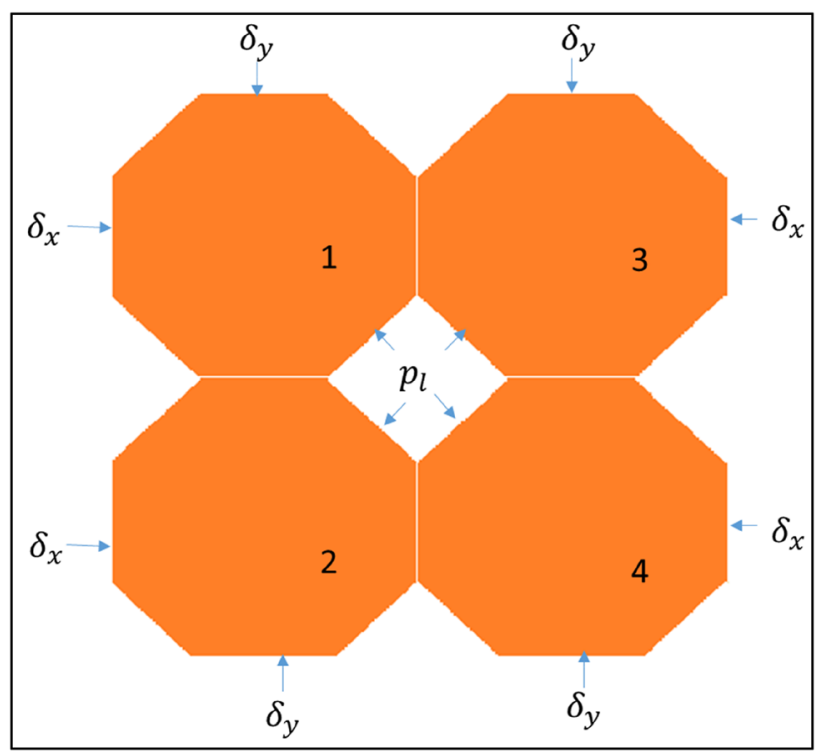

Fig. 1 Stress diagram of sandstone grain in pores 
During the development of an oilfield, due to the same buried depth and reservoir geological conditions, the conduction stress of a solid source remains substantially unchanged. Hence, the main stress variation comes from the change of fluid pressure.

In the experiment of variable confining pressure, the effective net stress changes through changes in the confining pressure. Essentially, the change of confining pressure is achieved by changing the stress loaded on the rubber sleeve of the core holder, thus adjusting solid source conduction stress and changing the effective net stress. Nonetheless, the method's conditions to change net stress change are different from the actual situation of oilfield development. Such difference between the experiment and oilfield development results in a large deviation between the experimental results and the field production, thus deteriorating the applicability of the experimental results.

\section{Analysis on the limitation of the variable fluid pressure test method}

The variable fluid pressure test method changes the net stress of rock particles by changing the pore fluid pressure. Compared with the previous method, this method has an experimental process consistent with the situation in oilfield development and therefore can solve the problems in the variable confining pressure test method.

However, even though the variable fluid pressure test method in the standard provides a solution for the problem of similarity between the laboratory and the field, its experimental results are still limited due to the experimental methods and equipment.

The calculation method of effective net stress is given in Eq. 1. When the fluid pressure increases, the effective net stress gradually decreases. When $\delta$ is less than $p_{l} \varphi$, the effective net stress becomes negative, meaning that the net effective stress changes from compressive stress to tensile stress.
The straining process in the reservoir can be divided into three stages. In the first stage, when the effective net stress is expressed as the compressive stress and remains a large value, the solid particles will closely contact while the change of fluid pressure has little effect on the contact form of the particles. In the second stage, the increased fluid pressure compresses the solid particles. Under constant total volume, the pore volume increases so does the seepage capacity of the porous medium. At this time, as the fluid pressure increases, the seepage capacity slowly increases. In the third stage, as the fluid pressure increases, the compressive stress value becomes smaller, the relative position of solid particles changes and the seepage space increases. In this process, as the fluid pressure increases, the change in seepage capacity becomes faster. At the same time, as the fluid pressure continues to increase, the effective net stress is expressed as tensile stress, which leads to the opening and expansion of micro-fractures and even the generation of more micro-fractures.

According to the above analysis, the second and third stages have the greatest impact on the seepage capacity of the reservoir. When the effective net stress is expressed as the low-pressure stress value and as the tensile stress value, the core becomes highly stress sensitive. In the production process of high-pressure water injection, the variation range of formation net stress can cover the above two processes.

Figure 2 shows the structure of the experimental core holder. In the experiment, by injecting fluid into the annulus, the rubber sleeve is tightly pressed on the core surface and the metal plugs at both ends of the core. Meanwhile, the stress is loaded on the core surface through the rubber sleeve. In the experiment, when the fluid pressure is lower than the annulus pressure, the fluid can only flow through the core. Whereas when the fluid pressure is higher than annulus pressure, the rubber sleeve will open, leading to fluid channeling on the core surface or fluid leakage from the equipment port. As a result, the measured fluid flow will
Fig. 2 Schematic diagram of the core holder

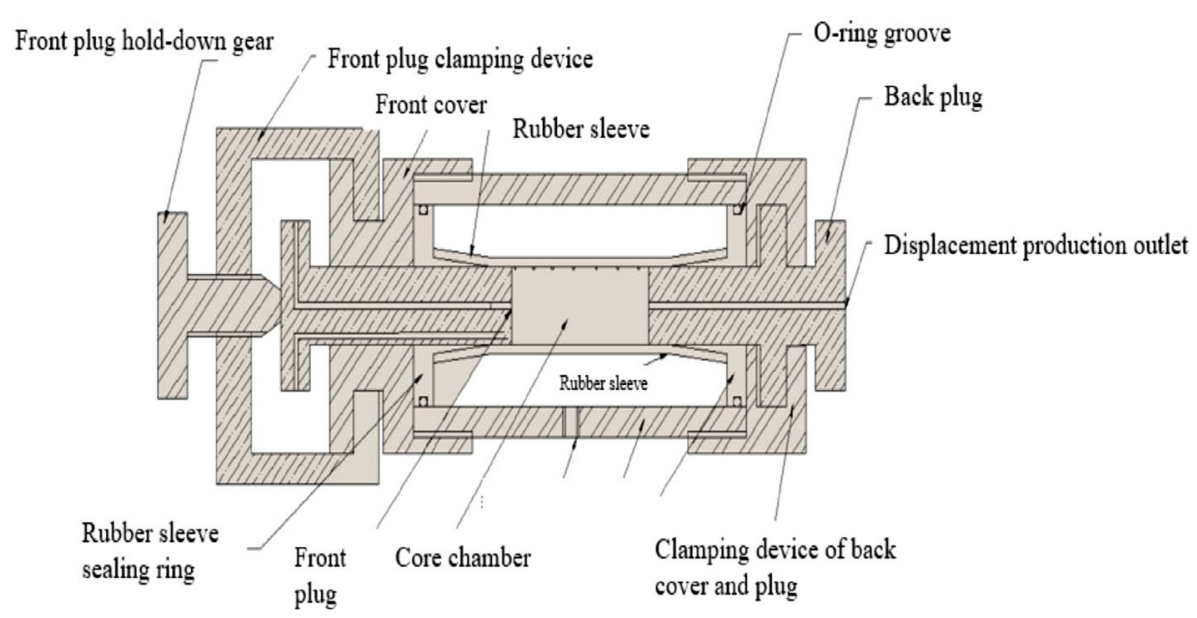

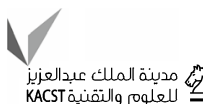
Springer 
be greater than the actual flow through the core. An accurate core flow rate can be obtained when the annulus pressure is greater than the fluid pressure. In other words, to complete the experimental process specified in the Standard SY / T5358-2010, the effective net stress of the experiment must be the compressive stress condition. The experiment can't be conducted accurately when the net stress is within the range of low-pressure stress and tensile stress.

To sum up, this experimental method can only partially characterize the influence of reservoir stress and strain on seepage. The same problem also exists in the variable confining pressure test method.

\section{Improved stress sensitivity test method for low permeability and tight reservoir}

The existing stress sensitivity test methods have certain limitations and therefore cannot fully and effectively reflect the relationship between the net stress of reservoir and the seepage capacity of porous media. It is of great significance to establish a new set of stress sensitivity measurement methods.

\section{The purpose of improving the stress sensitivity test}

The purpose of stress sensitivity measurement in industrystandard SY / T5358-2010 is to understand the process of pore throat deformation, fracture closure or opening when the net overburden stress changes and the extent to which the rock seepage capacity changes. Through the analysis of the standard experimental process, it can be found that the experimental methods provided by the current standard have great limitations and cannot meet the application needs of the oilfield. By establishing an improved stress sensitivity test method, this paper attempts to offset the shortcomings of existing standards and provide a new test and application method for determining the relationship between reservoir seepage capacity and stress.

\section{Introduction of improved stress sensitivity test method}

The new experimental method of stress sensitivity is improved based on variable fluid pressure. The improved experimental method realizes the change of the effective net stress range by introducing axial stress so that the change process of the effective stress can be measured from the compressive stress to tensile stress.

\section{Improved stress sensitivity experiment device}

The improved stress sensitivity test process consists of a displacement pump (or gas cylinder + pressure control system), confining pressure pump, axial pressure pump, backpressure control system, triaxial stress holder, pressure gauge system and flow measurement system. The flow chart of the experiment is shown in Fig. 3.

Since backpressure has a great influence on the calculation of gas seepage ${ }^{[2]}$, as suggested, the liquid is used as the flow medium in the experiment. In this experiment, a high concentration of standard brine was used as the experimental fluid.

The core of the experimental process is a triaxial stress holder that can load axial stress while loading confining pressure. Confining pressure pump loads a constant confining pressure for the triaxial stress holder; the axial pressure pump loads a constant axial pressure for the triaxial stress holder; the backpressure control system can provide stable backpressure for the experiment. The pressure measurement system measures the pressure difference between the two ends of the core. Through the pressure difference
Fig. 3 Improved equipment diagram of the stress-sensitive experiment

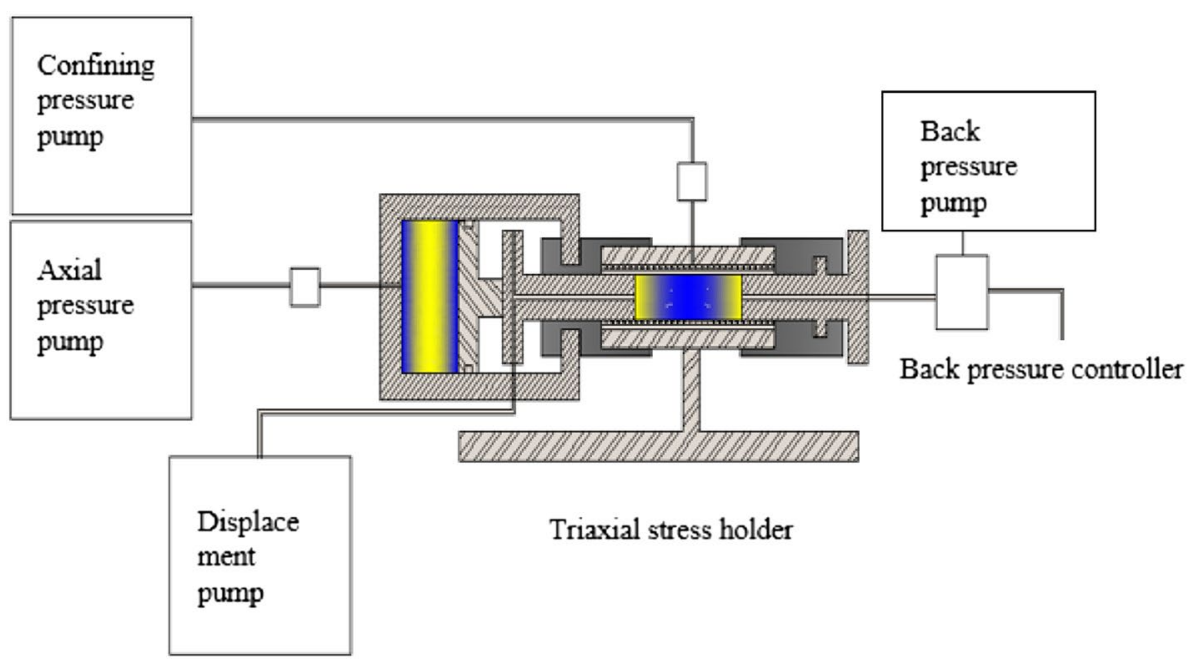


measurement and flow measurement results, the core seepage capacity under different experimental conditions can be calculated.

In the experiment, the confining pressure and axial pressure remain constant. The confining pressure simulates the stress perpendicular to the flow direction, while the axial pressure simulates the stress in the flow direction. By loading axial stress, the net stress of rock is tensile stress when the fluid pressure is lower than the confining pressure. Under a certain displacement rate, the net stress of rock particles can be changed by changing the backpressure and the fluid pressure in the pores.

\section{Experimental methods}

According to the improved process and requirements of the stress sensitivity experiment, the specific experiment steps are designed as follows (Fig. 4).

1. Core preparation: drill core from the reservoir, wash oil and let it dry and then saturate the dried core with formation water.

2. Put the core into the holder with the confining pressure loaded to $30 \mathrm{MPa}$.

3. Conduct water flooding at a velocity slower than the critical velocity of velocity sensitivity. Meanwhile, observe the injection pressure. If the injection pressure is greater than $3 \mathrm{MPa}$, reduce the injection speed to keep the injection pressure at about $3 \mathrm{MPa}$ and stop the injection. Take this flow rate as the experimental flow rate.

4. Load the axial pressure based on the need of the experiment. Take $5 \mathrm{MPa}$ as the gradient until the permeability changes suddenly.

5. Conduct displacement based on the set experimental flow rate.

6. Set the experimental back pressure as $0,5 \mathrm{MPa}, 10 \mathrm{MPa}$, $15 \mathrm{MPa}, 20 \mathrm{MPa}$ and $25 \mathrm{MPa}$. Keep each back pressure constant for more than $30 \mathrm{~min}$ and record the stable displacement pressure. When the inflection point appears, the backpressure experiment point can be added near the inflection point.

7. Calculate the permeability under different backpressure and draw the relationship curve between permeability and average fluid pressure. The curve is the experimental curve of core stress sensitivity.

In the experiment, the loading of axial stress should be combined with the actual stress condition of the reservoir and the demand for reservoir development. Because of the directionality of reservoir stress, the axial stress loading values in different directions are also different. When measuring the seepage capacity in the direction of minimum principal stress, the axial pressure should be less than the confining pressure. When measuring the permeability in the
Fig. 4 Main process node diagram of the experiment

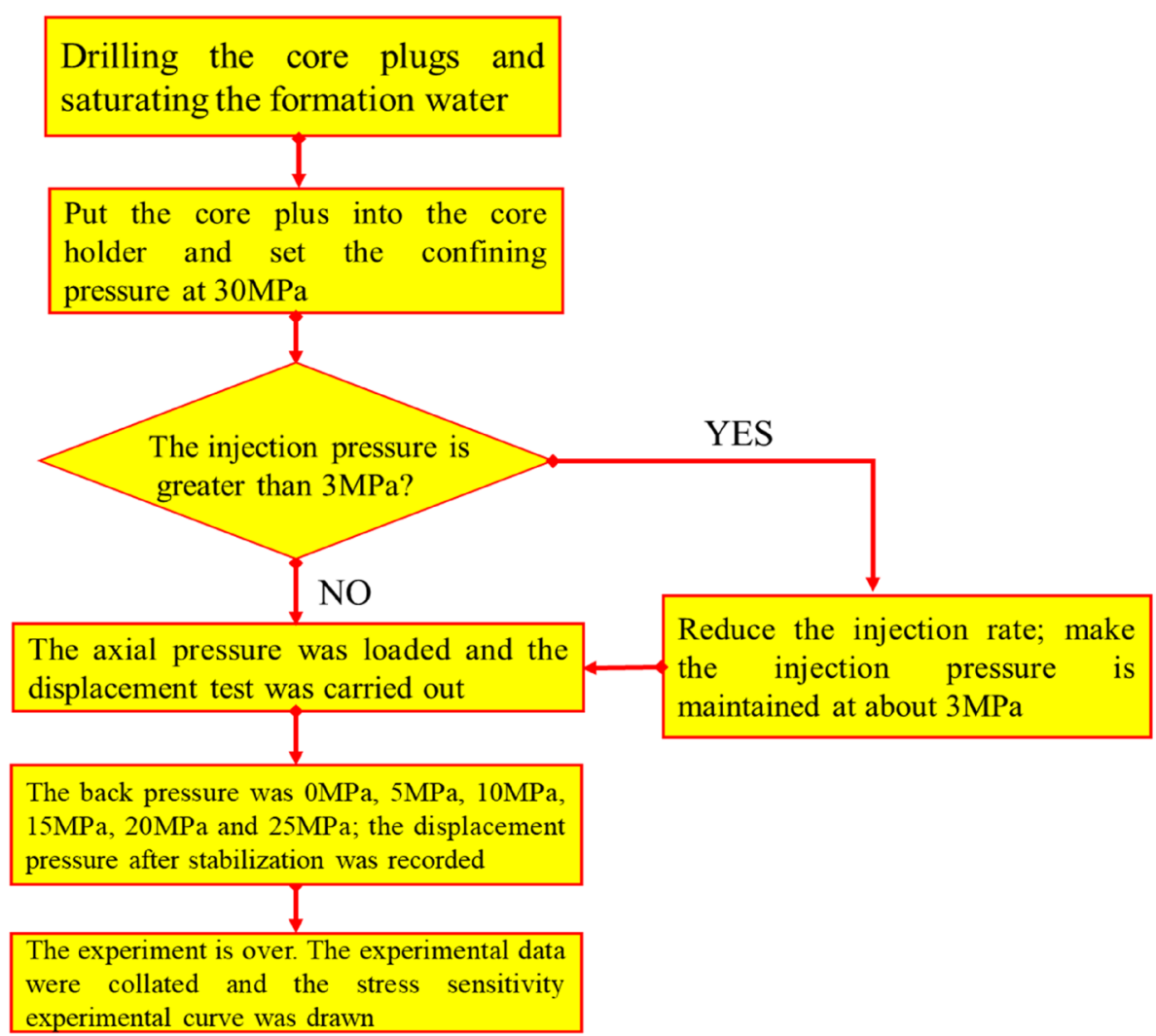


direction of the maximum principal stress, the axial stress should be greater than the confining pressure.

\section{Mechanism and Application}

\section{Effect of axial stress in the experiment}

In this experiment, the effective net stress is adjusted by loading axial stress. Take sandstone as an example and analyze the experimental mechanism.

Sandstone is composed of sandstone particles and cement. with a negligible volume of cement, it can be considered as the accumulation of particles of different sizes in different ways. With these particles simplified into spherical particles, the sandstone core is an accumulation body composed of spheres of different sizes. Next, the solid source stress of spherical particles is analyzed according to the particle accumulation mode in Fig. 5.

Figure 5 shows the stress decomposition of particle 3 in this stacking mode. In the figure, the longitudinal stress represents the axial solid source conduction stress and the stress on both sides represents the solid source conduction stress perpendicular to the flow direction.

In the figure, particles 1 and 2 are subjected to axial stress with an equal magnitude but opposite direction with a value of $\delta_{y}$. The radial stresses of particles 3 and 4 are equal in magnitude but opposite in direction with a value of $\delta_{x}$.

Particle 3 is mainly affected by $\delta 1, \delta 2$ and $\delta x$. In the longitudinal direction, $\delta 1$ and $\delta 2$ can be decomposed into $\delta 4$ and $\delta 5$. The two forces are equal in value but opposite in direction and their resultant force is 0 . In the horizontal direction, $\delta 1$ and $\delta 2$ can be decomposed into $\delta 3$.

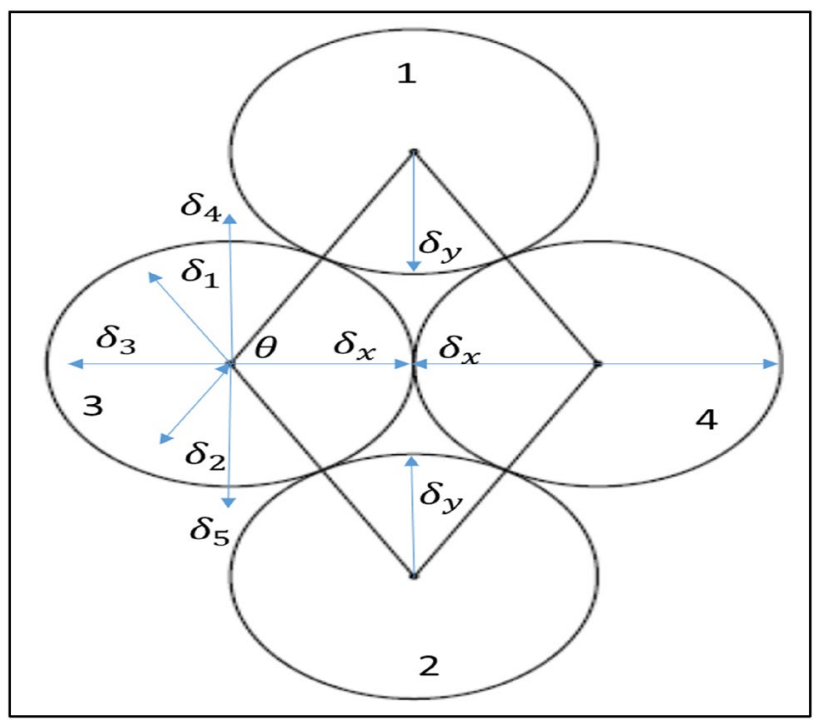

Fig. 5 Stress decomposition diagram of sandstone grain $\delta_{3}=2 \delta_{y} \cot \theta$

Similarly, the axial stress causes particle 4 to be subjected to right stress with the same magnitude as that of $\delta 3$. The loading of axial stress results in tensile stress between particles 3 and 4 . The loading of axial stress functions as the pre-loading of tensile stress.

Figure 6 shows the stress diagram of four particles around a pore under axial stress and fluid stress. It can be seen that when the fluid stress is applied, the two pairs of particles are respectively subjected to the stress in opposite and outward directions. When the axial loading stress is applied, the two pairs of particles are respectively subjected to the compressive stress in the opposite and inward direction. The radial stress (x-direction) has an outward direction and is expressed as the tensile stress.

The measurement direction of permeability is the axial direction ( $\mathrm{Y}$ direction). The compression and expansion of axial pores mainly affect the radial flow and the influence on the axial permeability can be ignored. The above analysis indicates that in the experiment the influence of loading axial stress on core permeability is consistent with that of loading fluid pressure.

According to Eq. 2, the value of tensile stress produced by axial compression is related to the magnitude of axial stress and the contact mode of rock particles. For a fixed core or reservoir, under the same axial stress conditions, the resulting tensile stress is constant. For cores with different microscopic arrangements, the corresponding tensile stress can be expressed as:

$p_{g}\left(\delta_{y}\right)=f\left(\delta_{y)}\right.$

The measured core axial permeability under the axial stress of $\delta \mathrm{z}$ and fluid pressure $\mathrm{p}_{1}$ is consistent with that under 0 axial stress and $p l+(\delta z)$ fluid pressure.

When $\delta z$ is fixed, $f(\delta z)$ is a constant. The experimental results under this axial stress condition can be regarded as the results of the 0 axial stress curve shifted to the left (as shown in Fig. 7). Through calculation, the curve of loaded axial stress can be calibrated to the curve of unloaded axial stress.

According to the above analysis, if the abscissa of the experimental results corresponds to the field, the stress sensitivity curve of the site can be obtained.

In conclusion, by loading the axial stress, the preloading of fluid pressure is achieved. When the fluid pressure is lower than the confining pressure, the effective net stress can be expressed as tensile stress.

\section{Results of conventional stress sensitivity test}

In this study, four cores of the target reservoir were selected for the conventional stress sensitivity test. Specific core 
Fig. 6 Stress diagram of sandstone grains of loading fluid pressure(left) and axial stress(right)
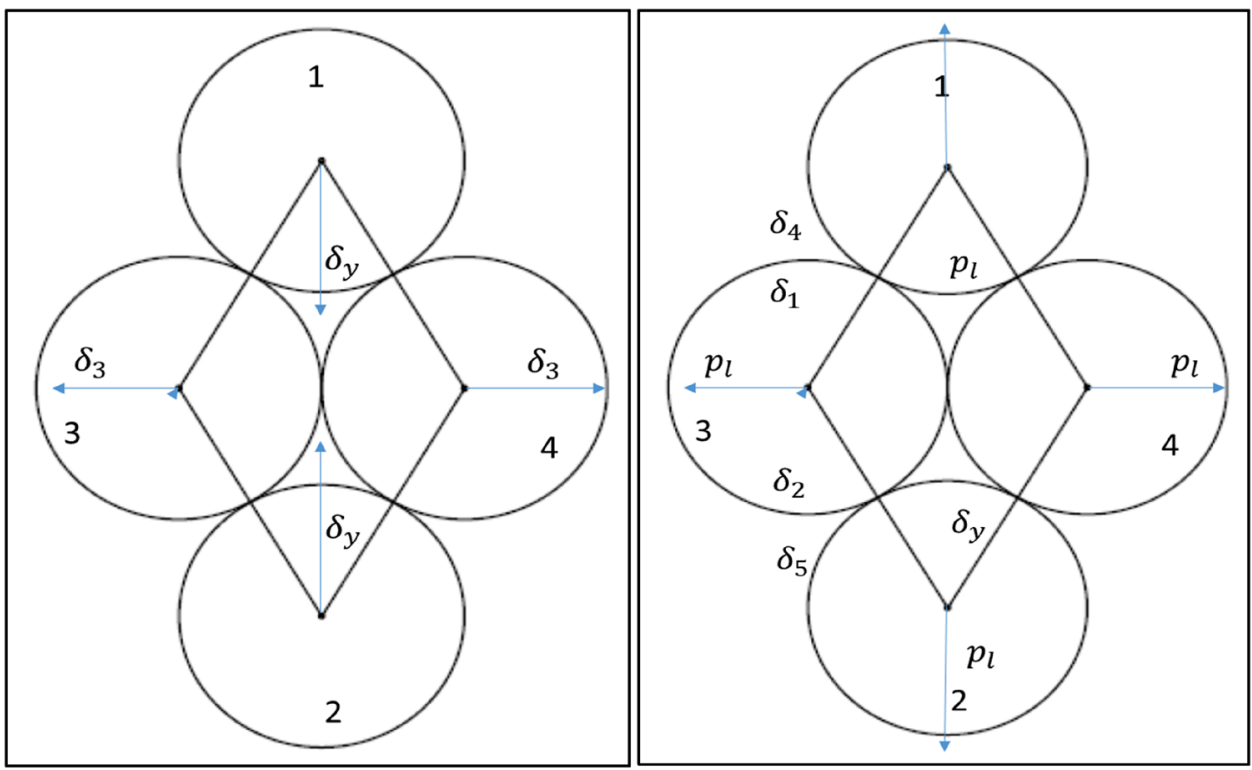

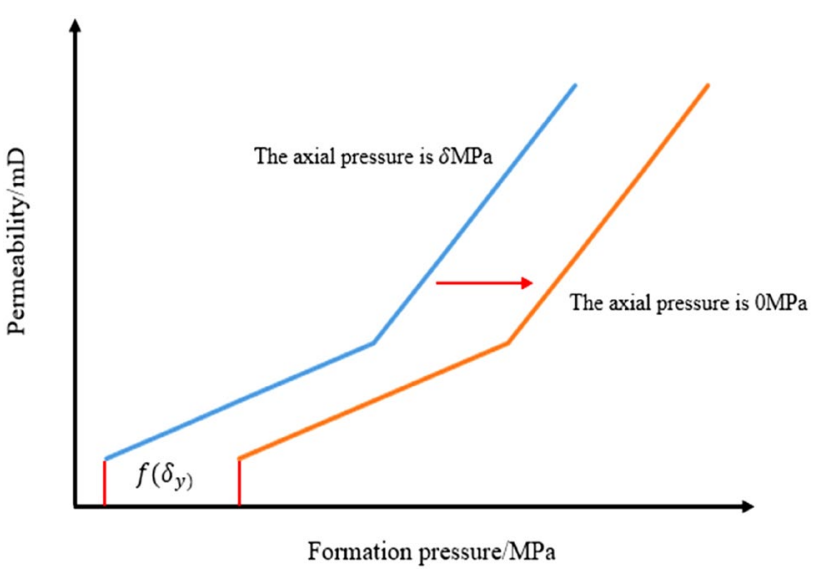

Fig. 7 Schematic diagram of the experimental curve and theoretical curve of zero axial stress

parameters and test results are shown in Table 1. According to the experimental method and evaluation standard in the petroleum industry standards, the stress sensitivity of the four cores is classified as medium to a strong level. Wherein, SK1 has the lowest permeability $(0.316 \mathrm{mD})$ and the highest stress sensitivity, with a permeability damage rate of $91.45 \%$. Whereas SK4 has the highest permeability
$(1.149 \mathrm{mD})$ and the lowest stress sensitivity, with a permeability damage rate of $33.50 \%$. In addition, the stress-sensitivity curves in the process of compression and decompression do not coincide, indicating the existence of the stress hysteresis effect (Fig. 8). A good exponential relationship exists between effective stress and normalized permeability $\left(\mathrm{R}^{2}>0.9\right)$.

\section{Application of stress sensitivity test method}

Four cores were selected from a tight reservoir in Ordos Basin. The improved experimental method was used to carry out a stress sensitivity measurement experiment for the cores with microfractures (Fig. 9). The basic data are shown in Table 1.

To illustrate the function of micro-fractures, before the experiment, micro-fractures were made inside the core by loading axial pressure to generate shear stress. In the experiment, the confining pressure is $50 \mathrm{MPa}$, which is equivalent to the overburden pressure and the axial pressure is $36 \mathrm{MPa}$.

From the experimental curves (Fig. 9), the stress sensitivity of the micro-fractured core can be divided into two stages in the process of fluid pressurization. In the first stage, the core permeability changes slightly and the stress sensitivity is weak, while in the second stage, the stress
Table 1 Conventional stress sensitivity evaluation result

\begin{tabular}{lllllll}
\hline Core & Length $/ \mathrm{cm}$ & Diameter $/ \mathrm{cm}$ & Perm $/ \mathrm{mD}$ & Poro/\% & $D / \%$ & Evaluation \\
\hline SK1 & 5.407 & 2.513 & 0.316 & 7.7 & 91.45 & Strong \\
SK2 & 5.048 & 2.517 & 0.574 & 11.2 & 85.36 & Strong \\
SK3 & 5.514 & 2.521 & 0.766 & 15.8 & 88.93 & Strong \\
SK4 & 5.442 & 2.513 & 1.149 & 16.3 & 33.50 & Medium \\
\hline
\end{tabular}


SK1

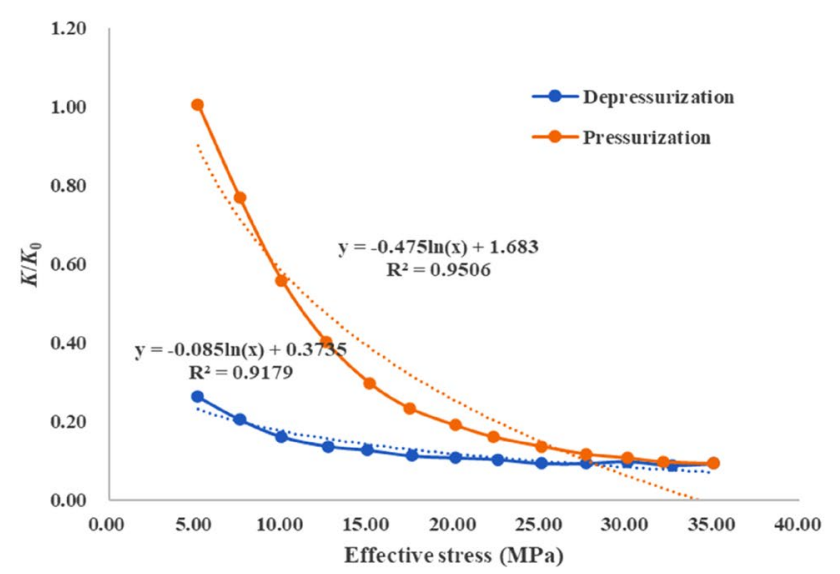

SK2

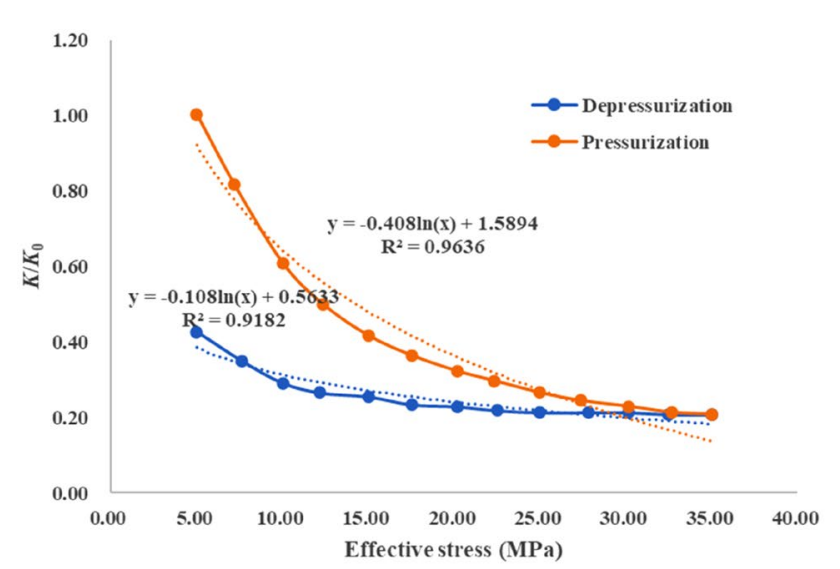

Fig. 8 Conventional stress sensitivity curve

sensitivity is enhanced. The fluid pressure corresponding to the intersection of the two stages is the re-open pressure of the micro-fracture.

According to the development performance of the selected block, when the wellhead water injection pressure is $25 \mathrm{MPa}$, the injection volume increases sharply, indicating that the microfracture re-open pressure of the reservoir is $25 \mathrm{MPa}$ in terms of wellhead pressure. The wellhead pressure can then be converted into 48MPA bottom hole pressure according to the well depth of $2300 \mathrm{~m}$. Take SK1 core data as an example (Fig. 10). The initial pressure in the experiment is $0 \mathrm{MPa}$. According to the bottom hole pressure, the curve is shifted to the right to obtain the stress sensitivity curve of the reservoir. Additionally, to compare the difference between the new tight sandstone stress sensitivity test method and the traditional stress sensitivity test method, the SK4 core is taken as an example for analysis. From Fig. 11, the stress sensitivity intervals characterized by the two methods can be found different. The traditional method can only characterize the
SK3

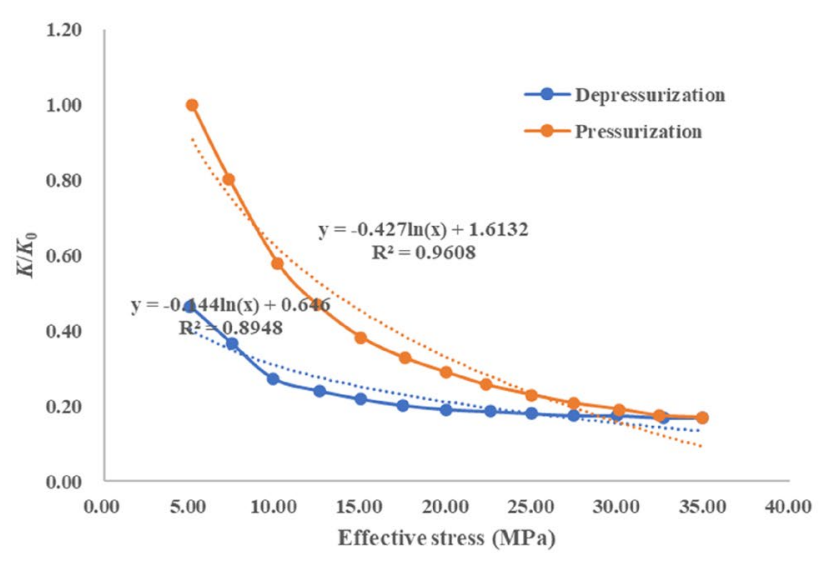

SK4

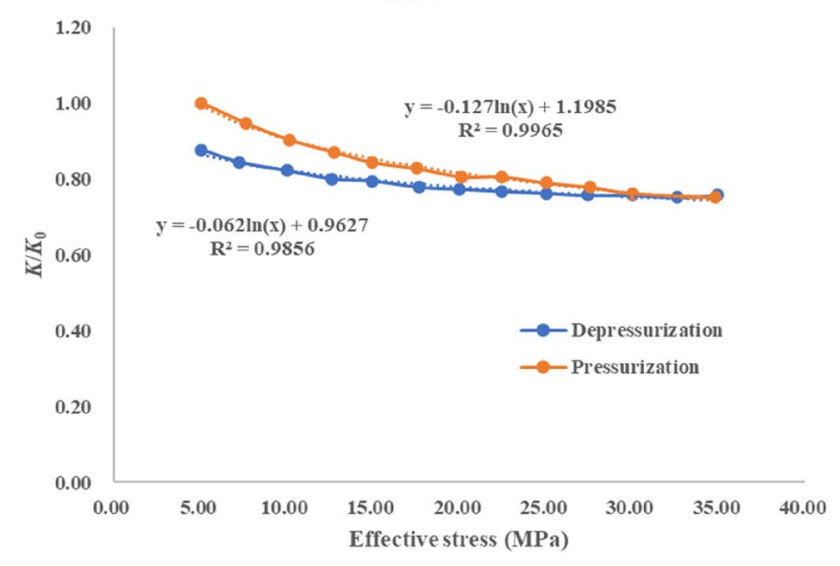

Fig. 8 (continued)

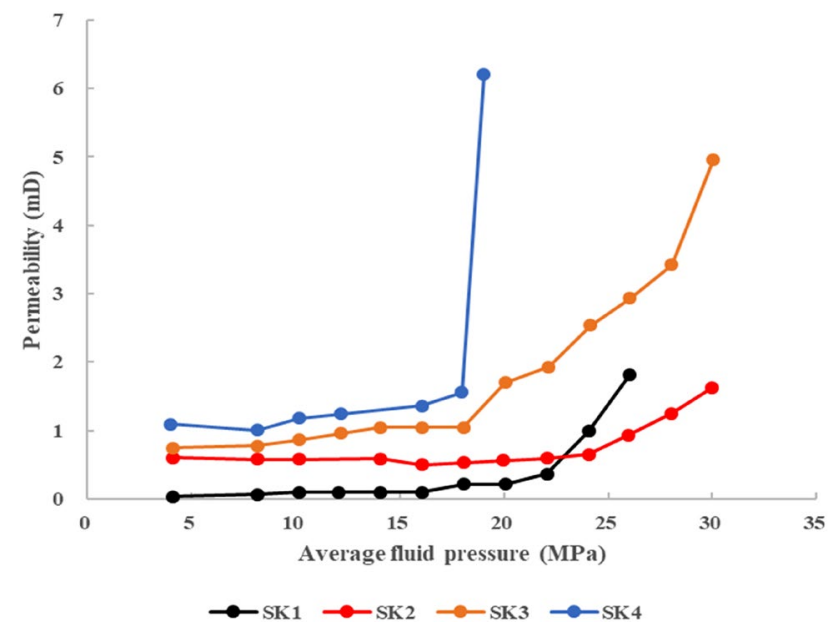

Fig. 9 Stress sensitive curve of four sandstone cores 


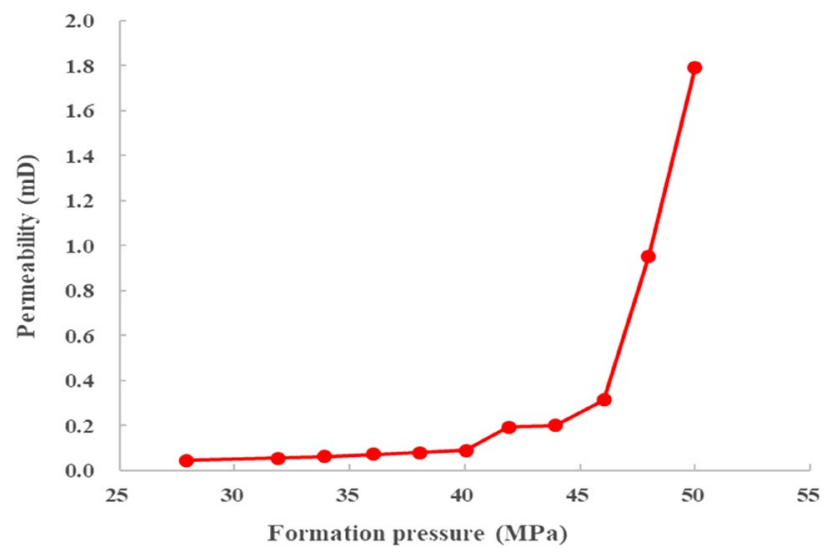

Fig. 10 Stress curve obtained after curve translation of sandstone core SK1

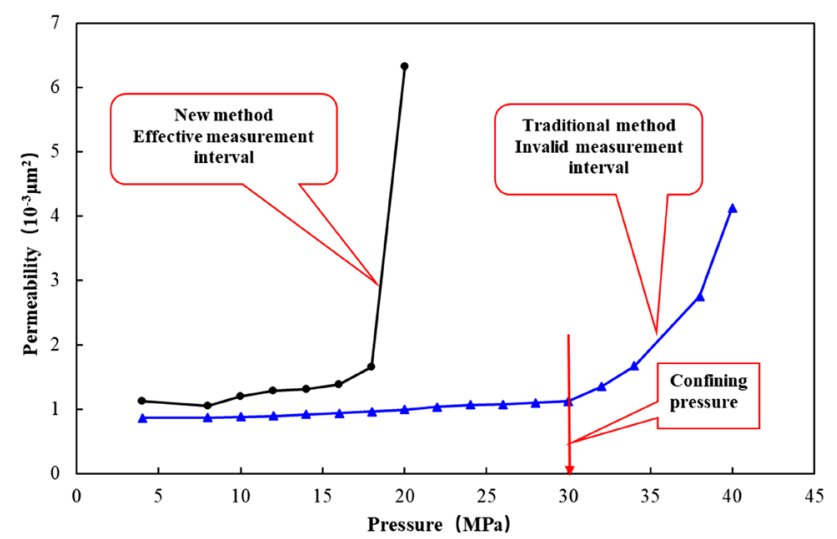

Fig. 11 Comparison of test results between the new method and the traditional method (SK4)

stress sensitivity before the reopening of fractures, while the new method can characterize the permeability stress sensitivity both before and after the reopening.

If the traditional experimental method is used to test the stress sensitivity of tight sandstone, when the fluid pressure is higher than the ring pressure, the rubber sleeve deforms and the fluid flows along the gap between the rubber sleeve and the core. As a result, the flow capacity at this time cannot illustrate the flow capacity inside the core. When the fluid pressure is greater than the ring pressure, the micro-fracture reopens. However, the stress sensitivity under high-pressure displacement conditions cannot be characterized by traditional equipment. On contrary, the new experimental method increases the axial stress, which can increase the fluid pressure inside the core, such that the fracture can be reopened even when the ring pressure is greater than the fluid pressure. Meanwhile, the measured data at this time characterizes the percolation capacity of the core itself.

\section{Conclusion}

1. By loading radial stress, a set of experimental methods for reservoir stress sensitivity is established, which changes the effective net stress range of the conven

2. tional variable back pressure experimental method and is of great significance to the application of stress sensitivity experimental data in oil development.

3. The main mechanism of the radial stress loading is to use the axial stress to pre-load a fixed value of the tensile stress in the radial direction, thus equivalently expanding the value of the fluid pressure.

4. By translating the resulting curve measured by this method, the stress sensitivity curve of the reservoir can be obtained.

5. The experimental data can be corrected and the benchmark permeability of the reservoir can be determined by the data in the process of field water injection.

6. The stress sensitivity of the micro-fracture reservoir during water injection can be divided into two parts. Before the re-opening of the fractures, the stress sensitivity is weak, but after the re-opening, the stress sensitivity becomes stronger.

Acknowledgements We gratefully acknowledge the financial supports from the National Science and Technology Major Project (2017ZX05013-001, 2016ZX05048).

Funding The author(s) disclosed receipt of the following financial support for the research, authorship, and/or publication of this article: This paper is sponsored by the National Science and Technology Major Project (2017ZX05013-001, 2016ZX05048).

\section{Declarations}

Conflict of interest The authors declare that they have no conflict of interest.

Open Access This article is licensed under a Creative Commons Attribution 4.0 International License, which permits use, sharing, adaptation, distribution and reproduction in any medium or format, as long as you give appropriate credit to the original author(s) and the source, provide a link to the Creative Commons licence, and indicate if changes were made. The images or other third party material in this article are included in the article's Creative Commons licence, unless indicated otherwise in a credit line to the material. If material is not included in the article's Creative Commons licence and your intended use is not permitted by statutory regulation or exceeds the permitted use, you will need to obtain permission directly from the copyright holder. To view a copy of this licence, visit http://creativecommons.org/licenses/by/4.0/. 


\section{References}

Boer R (1992) Development of porous media theories - A brief historical review[J]. Transp Porous Media 1992(9):155-164

Cao N, Lei G (2019) Stress sensitivity of tight reservoirs during pressure loading and unloading process. PETROLEUM EXPLORATION AND DEVELOPMENT 46:138-144

Chen Hao, Qin Yong,Li Guanzhong, et al (2018) Study on stress sensitivity of coal rock permeability based on pulse-decay method[J]. Coal Science and Technology. 2018, 46(6), 167-172.

Chen D, Pan ZhJ, Ye ZhH (2015) Dependence of gas shale fracture permeability on effective stress and reservoir pressure: Model match and insights. Fuel 139:383-392

Dazhen T, Zhejun P, Weiqiang H (2013) Characterization of the stress sensitivity of pores for different rank coals by nuclear magnetic resonance[J]. Fuel 111:746-754

Dou HE, Zhang HJ, Yao SG, Zhu D, Sun T, Ma ShY, Wang XL (2016) Measurement and evaluation of the stress sensitivity in tight reservoirs. PETROLEUM EXPLORATION AND DEVELOPMENT 43:1116-1123

Fanbao M, Li Xingfu P, Baud, et al (2020) Bedding anisotropy and effective stress law for the permeability and deformation of clayey sandstones[J]. Rock Mech Rock Eng 10(7):1-18

Li Chuanliang (2010) Are low permeability reservoir rocks strongly stress sensitive?[J]. Oil Drilling \& Production Technology. 32 (1),123-126.

Li Chuanliang, Zhu Suyang (2015) Misunderstanding of measuring methods of stress sensibility[J]. Lithologic Reservoirs. 27 (6), $1-4,29$.

Min Li, Wenlian X, Xiao G et al (2009) Laboratory study of the effective pressure law for permeability of the low-permeability sandstones from the tabamiao area, inner mongolia[J]. Chin J Geophys 52(6):1402-1413

Liang B, Jiang HQ, Li JJ, Gong ChCh, Jiang RY, Pei YL, Wei SM (2016) Flow in multi-scale discrete fracture networks with stress sensitivity. Journal of Natural Gas Science and Engineering $35: 851-859$
Liu YT, Yu P, Ding ZP (2018) Research on stress-sensitivity of fractured porous media. J Petrol Sci Eng 171:879-889

McKee CR, Bumb AC, Koenig RA (1988) Stress-dependent permeability and porosity of coal and other geologic formations. SPE Form Eval 3:81-91

Meng Y, Li ZhP, Lai FP (2015) Experimental study on porosity and permeability of anthracite coal under different stresses. J Petrol Sci Eng 133:810-817

National Energy Administration (2010) Formation damage evaluation by flow test: SY/T 5358-2010. Petroleum Industry Press, Beijing, p 2010

Sheng Yingshuai Hu, Qingxiong GH et al (2016) Evaluation on Stress Sensibility of Low Reservoir in Situ Conditions[J]. Acta Scientiarum Naturalium Universitatis Pekinensis 52(6):1025-1033

Xiao WL, Li T, Li M, Zhao JZ, Zheng LL, Li L (2016) Evaluation of the stress sensitivity in tight reservoirs. Pet Explor Dev 43:115-123

Zhao X, Yang Z, Wang Z et al (2020) An Experimental Study on Stress Sensitivity of Tight Sandstones with Different Microfractures[J]. Advances in Civil Engineering 2020:1865464

Yan Jian, Fu Meitao,Wang Rui,et al (2018) Influence of testing conditions on stress sensitivity of low permeability gas reservoir cores[J]. Journal of Xi'an Shiyou University(Natural Science Edition). 33(4), 55-60.

Zhang HY, He SL, Luan GH, Jiao ChY, Mo ShY, Liu H (2014) Quantitative study on stress sensitivity of ultra-low permeability fractured reservoirs. Chin J Rock Mechan Eng 33:3349-3354

Zhang JJ, Wei CT, Ju W, Yan GY, Lu GW, Hou XW, Kai Z (2019) Stress sensitivity characterization and heterogeneous variation of the pore-fracture system in middle-high rank coals reservoir based on NMR experiments. Fuel 238:331-344

Publisher's Note Springer Nature remains neutral with regard to jurisdictional claims in published maps and institutional affiliations. 